\title{
Methodology to determine failure characteristics of planar soft tissues using a dynamic tensile test
}

\author{
C. Jacquemoud ${ }^{\mathrm{a}, \mathrm{b}, *}, \mathrm{~K}$. Bruyere-Garnier ${ }^{\mathrm{a}}, \mathrm{M}$. Coret $^{\mathrm{b}}$ \\ ${ }^{a}$ Laboratoire de Biomécanique et Modélisation Humaine, INRETS, 25 Av. F. Mitterrand, case24, 69675 Bron Cedex, France \\ ${ }^{\mathrm{b}}$ Laboratoire de Mécanique des Contacts et des Solides, INSA de Lyon, Bât. 304, 69621 Villeurbanne Cedex, France
}

\begin{abstract}
Predicting the injury risk in automotive collisions requires accurate knowledge of human tissues, more particularly their mechanical properties under dynamic loadings. The present methodology aims to determine the failure characteristics of planar soft tissues such as skin, hollow organs and large vessel walls. This consists of a dynamic tensile test, which implies high-testing velocities close to those in automotive collisions. To proceed, I-shaped tissue samples are subjected to dynamic tensile tests using a customised tensile device based on the drop test principle. Data acquisition has especially been adapted to heterogeneous and soft biological tissues given that standard measurement systems (considered to be global) have been completed with a non-contact and full-field strain measurement (considered to be local). This local measurement technique, called the Image Correlation Method (ICM) provides an accurate strain analysis by revealing strain concentrations and avoids damaging the tissue. The methodology has first been applied to human forehead skin and can be further expanded to other planar soft tissues. The failure characteristics for the skin in terms of ultimate stress are $3 \mathrm{MPa} \pm 1.5 \mathrm{MPa}$. The ultimate global longitudinal strains are equal to $9.5 \% \pm 1.9 \%(\mathrm{Green}-\mathrm{Lagrange}$ strain), which contrasts with the ultimate local longitudinal strain values of $24.0 \% \pm 5.3 \%$ (Green-Lagrange strain). This difference is a consequence of the tissue heterogeneity, clearly illustrated by the heterogeneous distribution of the local strain field. All data will assist in developing the tissue constitutive law that will be implemented in finite element models.
\end{abstract}

Keywords: Soft tissue; Dynamic loading; Tensile test; Full-field strain; Failure characteristics

\section{Introduction}

Considering automotive collisions from 1993 to 1997, the most frequently and severely injured body region after the head appears to be the thorax $(28.7 \%)$ directly followed by the abdomen (15.6\%) (Lee and Yang, 2002). In frontal impact, a recent analysis of abdominal injuries (Yoganandan et al., 2000) indicates that the organs the most frequently subjected to critical injuries are the arteries $(14.2 \%)$ and the digestive system $(8.7 \%)$,

\footnotetext{
${ }^{*}$ Corresponding author. Laboratoire de Biomécanique et Modélisation Humaine, INRETS, 25 Av. F. Mitterrand, case24, 69675 Bron Cedex, France. Tel: +330472 1423 84; fax: + 330472142360 .

E-mail address: clementine.jacquemoud@inrets.fr (C. Jacquemoud).
}

ranked right after the liver and the spleen. Similarly, as soon as thoracic large vessels are involved, corresponding injuries tend to range from severe to fatal. Therefore, better understanding the injury mechanisms and predicting the injury risk for these organs present an important stake. However, the failure characteristics of such soft biological tissues under dynamic loadings are not clearly established yet. Concerning planar soft tissues, the mechanical properties already studied exclusively concern quasi-static loadings on rabbit skin (Lanir and Fung, 1974), human skin (Marcellier et al., 2001), bovine pericardium (Sacks, 1999) or canine pericardium (Choi and Vito, 1990), human internal carotid arteries (Stemper et al., 2005), porcine and human kidney capsule (Snedeker et al., 2005). Moreover, only the most recent studies (Snedeker et al., 2005; 
Stemper et al., 2005) reported failure characteristics and only the first one (Snedeker et al., 2005) concerns dynamic loadings. This lack of data is due to the difficulty in obtaining reliable measurements on soft biological tissues (Sanghavi et al., 2004). This difficulty is amplified when the tests are carried out in dynamic loading conditions up to failure and in high strain conditions.

A new dynamic tensile test methodology has been developed to determine the failure characteristics of planar soft tissues, such as skin, hollow organs and large vessel walls. The measurement technique has been chosen according to the tissues tested. Due to the heterogeneity of these tissues, a full-field strain measurement technique has been used to bring out possible

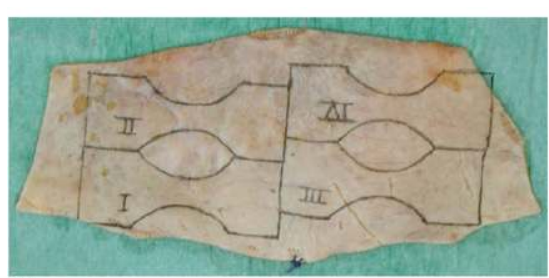

Cranial direction

$\uparrow$
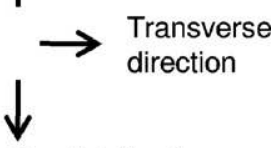

(a)

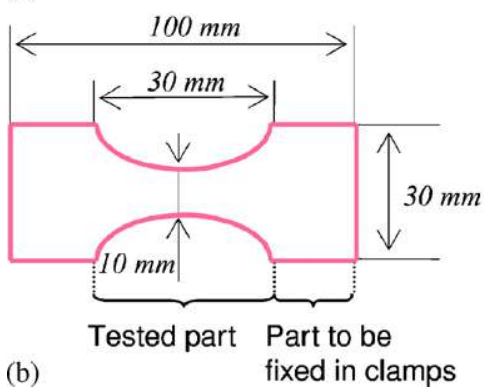

Caudal direction

Fig. 1. Skin preparation. (a) Sample disposition on the forehead skin piece. (b) I-shape sample dimensions. strain concentrations. In this paper are presented the methodology and the results on human skin.

\section{Methods}

\subsection{Preparation of skin samples}

A rectangular skin piece of roughly $200 \times 100 \mathrm{~mm}$ was removed from an 85-year-old male unembalmed Post Mortem Human Subject at the Rockfeller Medical University (Lyon, France). After removal, four I-shaped samples (Fig. 1) were cut along the transversal direction and kept in a physiological saline solution at $4{ }^{\circ} \mathrm{C}$ until testing (at least during $12 \mathrm{~h}$ ). The fascia and most of the hypodermis were removed from each sample so that only the epidermis and the dermis remain. Prior to testing each sample was covered by a random pattern of black dots made of mascara (Fig. 2a). This random pattern is needed for the measurement of strain fields using the Image Correlation Method (ICM) described later on. Before testing, the initial width $\left(l_{0}\right)$ and thickness $(e)$ of each sample were measured with a calliper.

\subsection{Tensile device for dynamic tests}

The extremities of the sample were attached to mechanical parts, respectively, named the upper clamp (UC) and the lower clamp (LC). In order to perform dynamic tensile tests, a new tensile device was designed to be adapted to an existing vertical drop bay (maximal high $=4 \mathrm{~m}$ ). The tensile device is divided into two parts: the main trolley, to which the UC is attached and the secondary trolley, to which the LC is attached. The secondary trolley is free to slide down (Fig. 3), simply

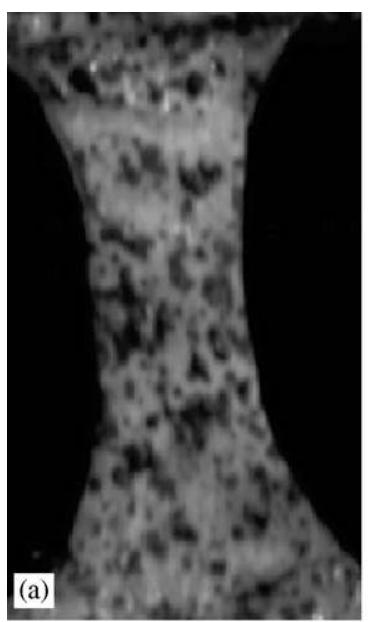

$\longmapsto 3 \mathrm{~mm}$

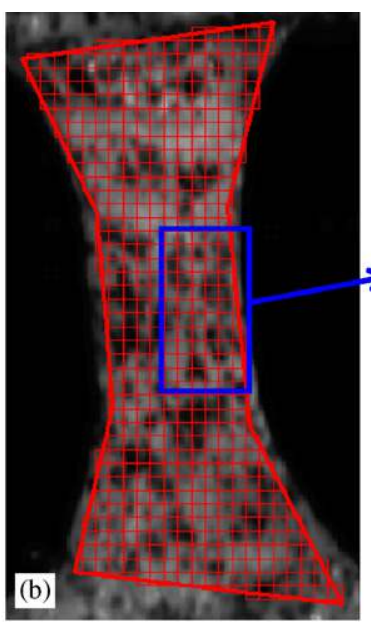

$\longmapsto 3 \mathrm{~mm}$

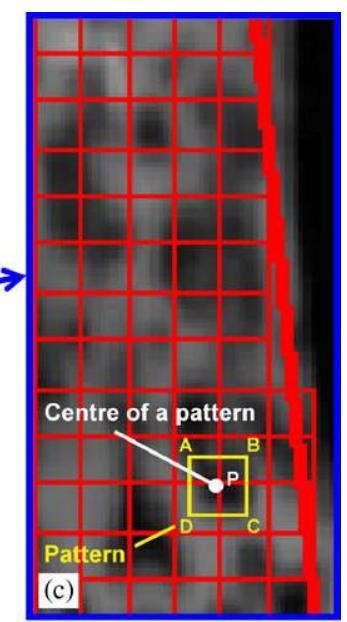

$\longmapsto 3 \mathrm{~mm}$

Fig. 2. Test RHD02: extract of the numerical image used by the ICM at $t=2 \mathrm{~ms}$. (a) The random pattern of black dots covering the sample surface. (b) The area of interest is defined in red on the sample image and is filled with a grid representing the centres of the patterns. (c) One of the 502 patterns $(\mathrm{ABCD})$ centred in $\mathrm{P}$. 


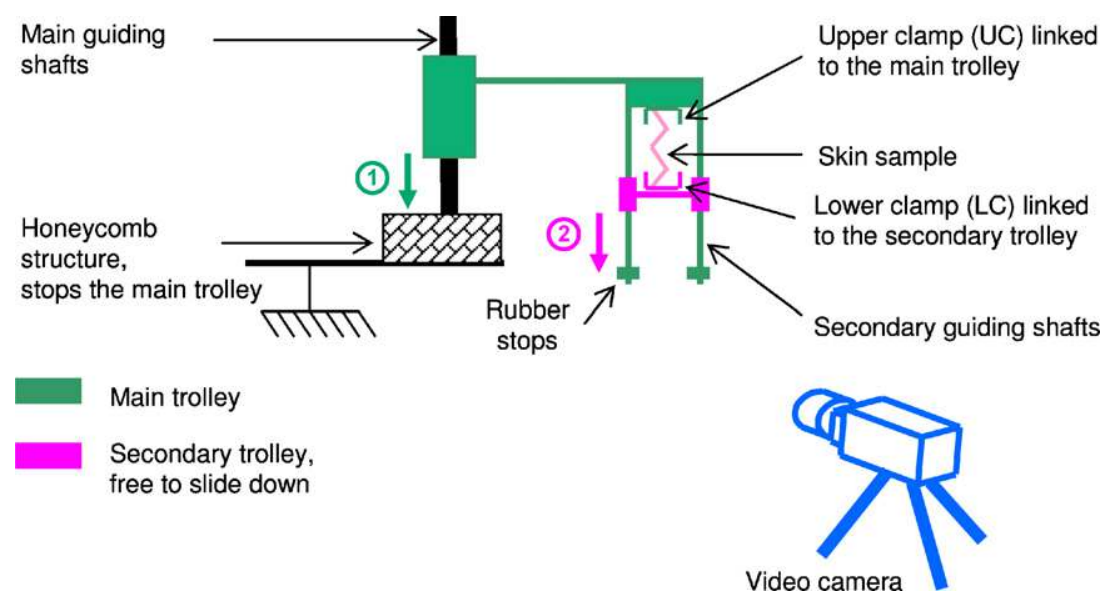

Fig. 3. Tensile device for dynamic tests on planar soft tissues.

linked to the main trolley by the sample. During a dynamic tensile test, all of the system (main trolley, secondary trolley and sample) slides down (step 1 in Fig. 3) until the main trolley is stopped by a honeycomb structure. From this time the secondary trolley follows its fall (step 2 in Fig. 3), loading the sample until failure.

Two parameters can be modified before the test: the initial velocity of the $\operatorname{LC}\left(V_{0}\right)$, which is defined by the height of the main trolley fall, and the weight $\left(M_{0}\right)$ attached to the LC. Each test was performed with $V_{0}=3 \mathrm{~m} / \mathrm{s}$ and $M_{0}$ equal to $1.8 \mathrm{~kg}$ (test RHD01), $1.77 \mathrm{~kg}$ (test RHD02), $1.58 \mathrm{~kg}$ (test RHD03), $1.39 \mathrm{~kg}$ (test RHD04). Data concerning the last three tests have been analysed and presented in this paper.

\subsection{Measurements}

The acceleration and the load of both UC and LC were recorded using standard measurement systems. The clamp-to-clamp displacement was measured by a linear variable differential transducer (LVDT) and called global sample elongation. All data were acquired at $10 \mathrm{kHz}$, and filtered according to SAEJ211 normalization (SAEJ211/1, Rev.Mach, 1995) with a Class $180 \mathrm{~Hz}$ filter. Global longitudinal strain was calculated from the global sample elongation measurement divided by the initial distance $\left(l_{0}\right)$ between the 2 clamps.

The experimental set-up was also equipped with a high-speed video camera. Images of the sample throughout the test were recorded at $1000 \mathrm{~Hz}$, which implies a $1536 \times 1024$ image size with a pixel size of $0.1 \times 0.1 \mathrm{~mm}^{2}$. The local strain field was measured by the ICM (more details on the method are in the Appendix A) using the Icasoft $^{\circledR}$ software (MguilTouchal et al., 1997, 1998). Displacement and strain calculations were performed inside an area of interest (defined on the sample in Fig. 2b) for each pattern of $0.9 \times 0.9 \mathrm{~mm}^{2}$. Results were available in more than 500 calculation points (centre of patterns) all over the area of interest with accuracy of $1 / 100$ pixel. The local sample elongation is defined as the difference between displacements measured at both extremities of the sample by the ICM.

The time history curves of the UC acceleration, the LC load and the global sample elongation as well as the stress-strain curves are plotted. The following failure characteristics are computed: ultimate stress, ultimate global strain and ultimate local strain (assumed to be the highest local strain before failure in the longitudinal direction).

\section{Results}

The time history curves of the UC acceleration, the LC load and the global sample elongation demonstrate 3 steps (Fig. 4a). Corresponding phenomena can be identified on the longitudinal local strain field measured by the ICM (Fig. 4b). Firstly, during the free fall of the entire system (up to $t=5 \mathrm{~ms}$ ), the sample is not loaded. No relative movement between the $\mathrm{UC}$ and the $\mathrm{LC}$ occurs, the LC load remains equal to zero and a low and constant local longitudinal strain field is obtained by the ICM. During this first step, the local sample elongation calculated by ICM appears similar to the global sample elongation measured by the LVDT on Fig. 5. Secondly, as soon as the skin starts to be stretched (from $t=5$ to $8 \mathrm{~ms}$ ), the global sample elongation and the LC load measurements increase. The local longitudinal strain field values also increase and the highest values are localized in specific areas on the sample (Fig. 4b). The local and global sample elongation values differ slightly at the end of this second step (Fig. 5). Thirdly, at the time of the maximal load $(t=8 \mathrm{~ms})$, the highest local longitudinal strain values are noticed in the area of the failure. These highest local longitudinal strains can reach twice the global longitudinal strain value. For instance, in test RHD02, the highest local strain values 

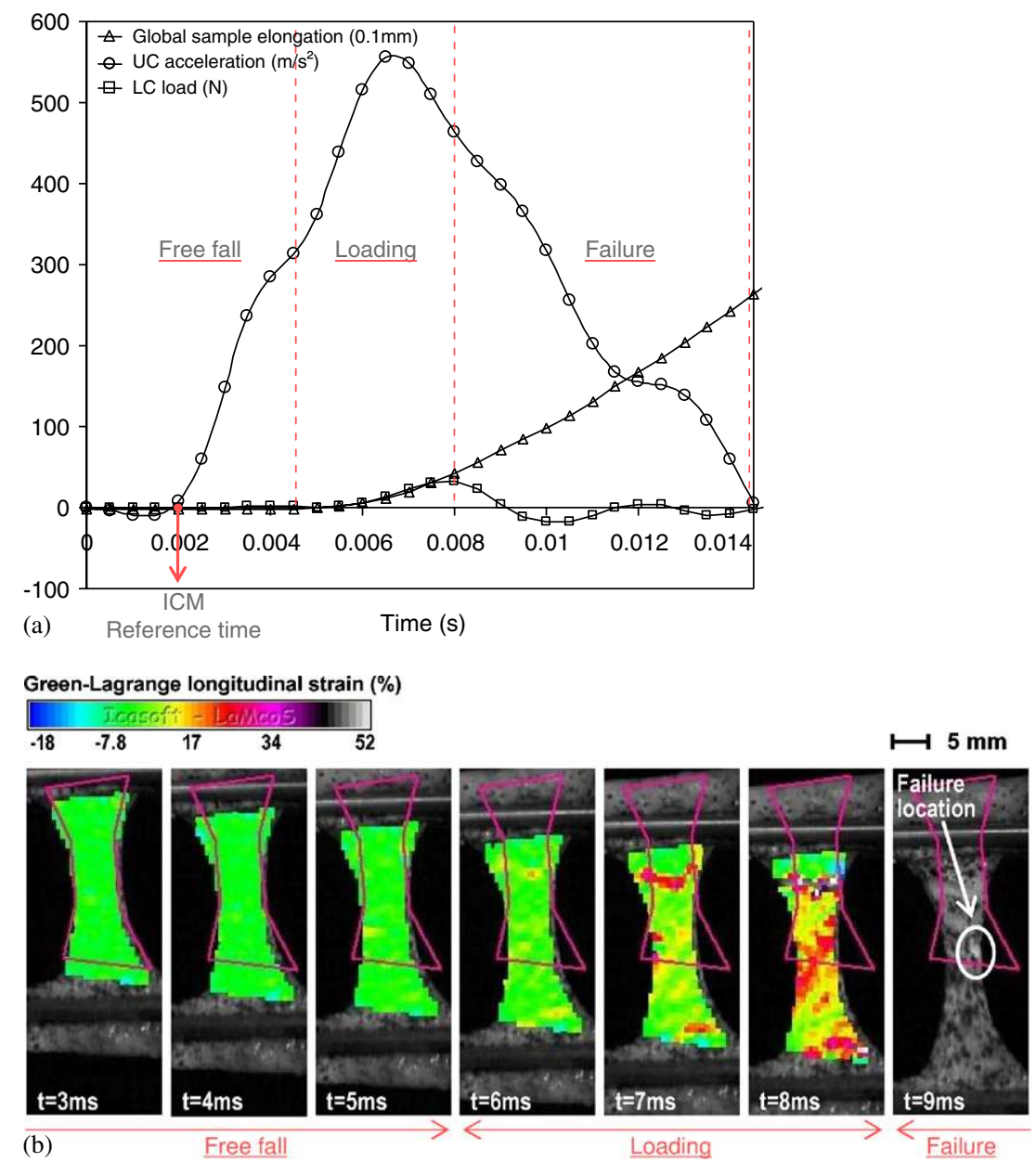

Fig. 4. Test RHD02: (a) the time history curves of the UC acceleration, the LC load and the global sample elongation. (b) The local strain field (Green-Lagrange longitudinal strain) calculated by the ICM.

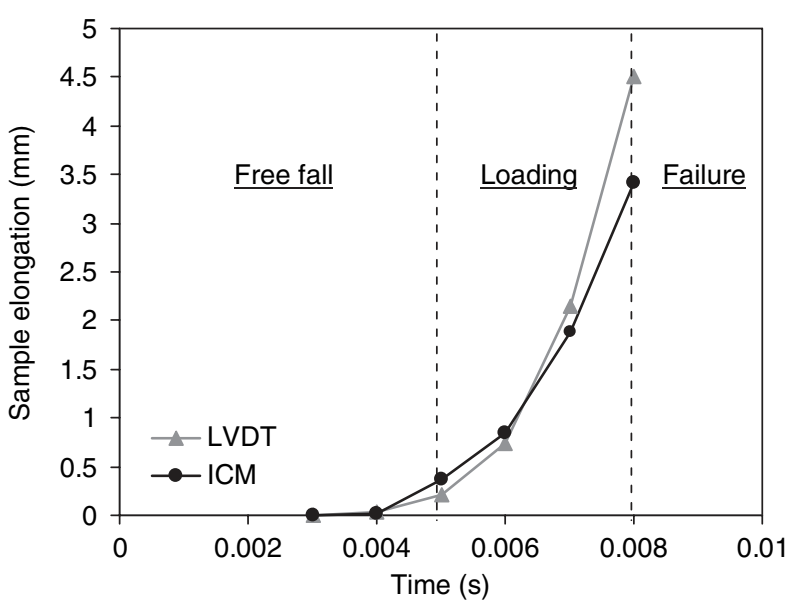

Fig. 5. Test RHD02: a comparison of the sample elongation measured locally by the ICM and globally by the LVDT.

are calculated to be $22 \%$ (Green-Lagrange strain) while the global longitudinal strains have a value of $11.6 \%$ (Green-Lagrange strain). On the opposite in both the transverse and the shear directions, the local strain field is homogeneous during the test: no strain concentration is noticed.

The stress-strain curves of test RHD02 (Fig. 6a) demonstrate that global and mean local longitudinal strain values are in good agreement. Each plot from Fig. $6 \mathrm{~b}$ to $\mathrm{g}$ represents the spatial distribution of the local longitudinal strains all over the sample calculated by the ICM for one image. One plot corresponds to one image, from $t=3$ to $8 \mathrm{~ms}$. The mean local longitudinal strain values indicated on each of the 6 plots are also those plotted on Fig. 6a as mean local longitudinal strain values (ICM). The standard deviations of these 6 distributions lie from $1.5 \%(t=3 \mathrm{~ms})$ up to $10.1 \%$ before failure $(t=8 \mathrm{~ms})$, this illustrates the increasing heterogeneity of the longitudinal strain field during the elongation. This heterogeneity also clearly appears when the longitudinal strains are represented all over the sample with a colour scale (Fig. 4b): the apparition of strain concentrations (Fig. 4b) close to failure is related to the increasing standard deviation of the spatial longitudinal strain distributions (Fig. 6b-g). Finally this heterogeneity is markedly higher than the ICM noise. 


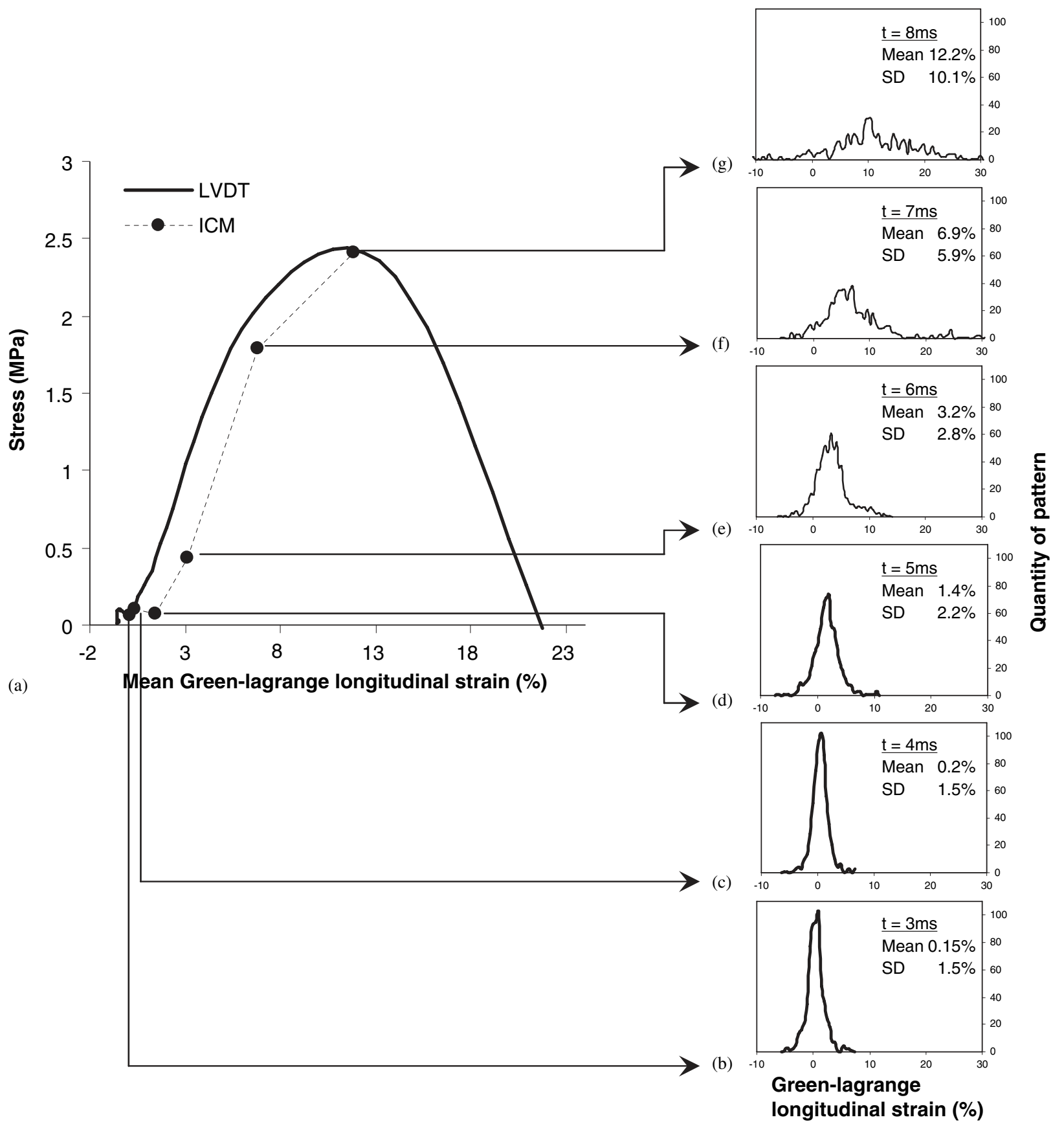

Fig. 6. Test RHD02: (a) the mean local strains (ICM) are compared to the global strains obtained from the LVDT values. (b)-(g) The spatial distribution of the local strain values all over the sample calculated by the ICM. One plot corresponds to one image, from $t=3$ to 8 ms. The mean local strain value is indicated for each distribution as well as the standard deviation (SD).

Fig. 7 represents stress-strain curves for the 3 tests, including longitudinal, transverse and shear strains measured by the ICM. Concerning the failure characteristics for these 3 analysed samples, the ultimate stress of the skin was $3 \pm 1.5 \mathrm{MPa}$. The ultimate global longitudinal strains were equal to $9.5 \% \pm 1.9 \%$ (GreenLagrange strain). The ultimate local longitudinal strains were $24.0 \% \pm 5.3 \%$ (Green-Lagrange strain).

\section{Discussion}

The present methodology consists of undertaking dynamic tensile tests on planar soft tissues with a tensile device based on the drop-test principle. Failure characteristics are though deduced for the skin at high strain rate (mean value $55 \mathrm{~s}^{-1}$ ). The ICM has been used to perform full-field strain measurements once the 

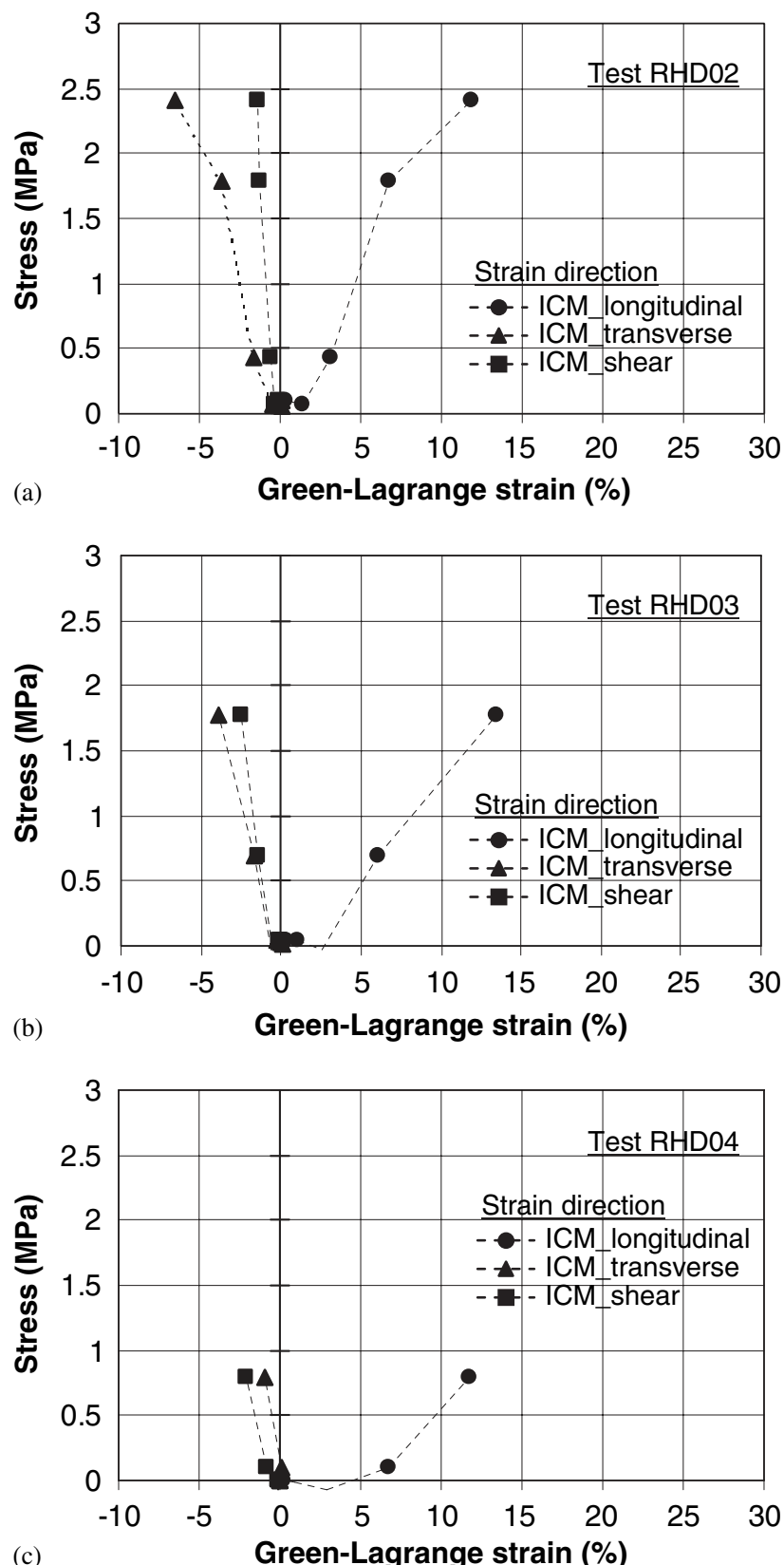

Fig. 7. The stress is plotted against the 3 components (longitudinal, transverse and shear) of the local strain field for (a) tests RHD02, (b) test RHD03 and (c) test RHD04. Strain values are the mean local values calculated by the ICM in each direction.

technical problems (Sanghavi et al., 2004) related to using this measurement technique on soft biological tissues had been solved. Therefore each test provides loading data associated to both global standard strain measurements and local non-contact strain measurements.

The reliability of the ICM has been established by comparing all measurement techniques. Zero force on the load cell corresponds to zero strain on the ICM measurements. The load and acceleration time history curves are divided in 3 steps as well as the ICM results. The local sample elongation calculated by ICM stays similar to the global sample elongation measured by the LVDT until $5 \mathrm{~ms}$, then the global value increase more quickly. Unlike the LVDT, which gives clamp-to-clamp displacements, the ICM measures displacements between points on the surface of the sample. The ICM presents the advantage to provide with true strains on the surface of the sample, therefore the difference between the local and the global elongation values corresponds to the slipping effects. Using the ICM, alllongitudinal transverse and shear strain values are calculated. Poisson's effect is though revealed by the local transverse strains. The local shear strains being not null the tensile test is not perfect. A non-perfect geometry of the sample, a non-perfect loading or slippage could lead to the creation of shearing, but this effect is quantified and remains very low.

The ICM, as a full-field strain measurement technique provides the entire strain field (longitudinal, transverse and shear components) in more than 500 calculation points (centre of patterns) spread every $0.9 \mathrm{~mm}$ all over the sample. On the opposite, only the longitudinal clamp-to-clamp displacement is measured by a standard LVDT. Therefore, measuring the longitudinal strain field in any points of the sample has the advantage to point out the heterogeneity of tissues such as skin. This heterogeneity is quantified by the standard deviation measured on the spatial distributions of local strains. Moreover, very high local strain values appear in some patterns close to the end of the test; this enables us to measure accurate strain values close to the failure location and time. The ICM is also defined as a non-contact measurement technique; its use avoids damaging such a material easily deformed during sample preparation.

The ultimate tensile stress of the skin calculated in this dynamic study is close to the literature values, obtained from quasi-static tensile tests. Silver et al. (1992) reports an ultimate tensile stress from 2 to $15 \mathrm{MPa}$ depending on the testing protocol and the skin removal site. More precisely for the forehead skin, an ultimate stress value of 4.6 MPa (average value for adults) was extrapolated from global data of a tensile test (Yamada, 1970). However, the ultimate tensile strain reported in the literature for the forehead skin in quasi-static tests is $54 \%$ (Yamada, 1970). This value is about twice higher than the ultimate local longitudinal strain calculated in this dynamic study.

More tests are now to be conducted on human skin to allow for statistical evaluation. The failure characteristics of skin tissue will be used to develop a damage predictive model in relation to the tissue microstructural parameters. The failure characteristics of skin tissue will also contribute to the implementation of new 
constitutive laws in finite element models dedicated to automotive collisions. Finally, this methodology will be further expanded to other planar and fibrous soft tissues, including abdominal and thoracic organ walls.

\section{Appendix A. The image correlation method (ICM)}

Mainly developed by Sutton et al. (Cheng et al., 2002; Schreier and Sutton, 2002; Sutton et al., 1983, 1986), the Image Correlation Method requires grey level images of the sample during a test. The sample surface has to be covered by a random pattern of black dots so that the displacement and strain field determination can be carried out according to the following principles:

- Displacement and strain fields are measured in an area of interest defined on the sample surface by the user (Fig. 2b). This area is then divided into groups of pixels called patterns (Fig. 2c). The correlation calculations are performed for each pattern with the hypothesis that the displacement field is homogeneous inside a pattern.

- The image of the sample is defined by a discrete function representing the grey levels of each pixel. The values of this function lie from 0 to 255 . In doing so, a discrete function $g(u, v)$ is attributed to the "initial image" representing the sample before distortion. This discrete function is transformed to $g^{*}\left(u^{*}, v^{*}\right)$ in order to define the "final image" of the deformed sample. The theoretical relationship between these two discrete functions is given by Eq. (1):

$g^{*}\left(u^{*}, v^{*}\right)-g(u+D U(u, v), v+D V(u, v))=0$,

where $D U(u, v)$ and $D V(u, v)$ represent the 2 components of the displacement field for a pattern (Fig. 8).

- The displacement field for each pattern is chosen homogenous and bilinear in $u$ and $v$ (Eq. (2)).

$$
\begin{aligned}
& D U(u, v)=a_{u} u+b_{u} v+c_{u} u v+d_{u}, \\
& D V(u, v)=a_{v} u+b_{v} v+c_{v} u v+d_{v} .
\end{aligned}
$$

In a recent study (Lu and Cary, 2000), different types of displacement fields have been tested in order to analyse more complicated deformation state.

- The determination of all terms (rigid body, strains) is made by a bilinear interpolation of the $g^{*}$ function and by minimization of the cross-correlation coefficient defined in Eq. (3):

$$
C=1-\frac{\int_{\Delta M} g(u, v) g^{*}\left(u^{*}, v^{*}\right) \mathrm{d} u \mathrm{~d} v}{\sqrt{\int_{\Delta M} g(u, v)^{2} \mathrm{~d} u \mathrm{~d} v \int_{\Delta M} g^{*}\left(u^{*}, v^{*}\right)^{2} \mathrm{~d} u \mathrm{~d} v}},
$$

where $\Delta M$ is the area of the pattern in the initial image.

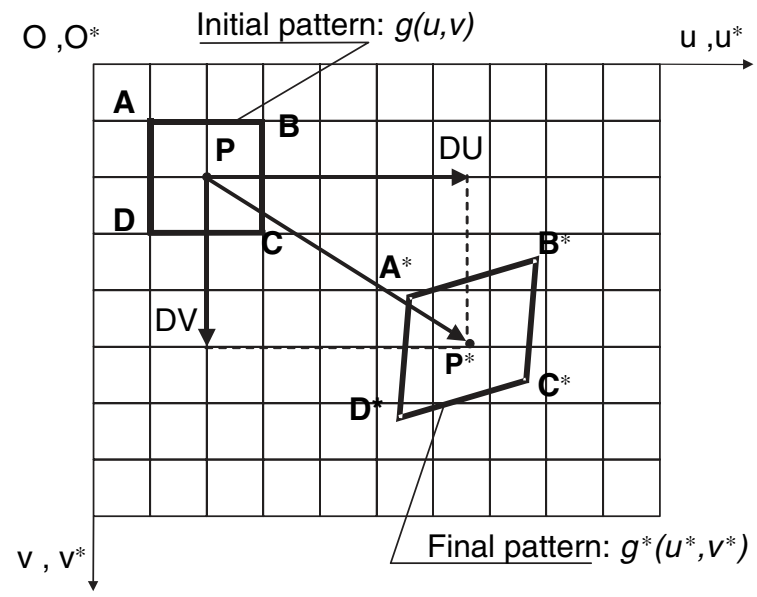

Fig. 8. The pattern evolution from initial image to final image. For a better understanding, the initial $(\mathrm{ABCD})$ and final $\left(\mathrm{A}^{*} \mathrm{~B}^{*} \mathrm{C}^{*} \mathrm{D}^{*}\right)$ patterns are represented on the same reference system.

- The displacement field values are given at the centre of each pattern. Strain field values can also be computed using the transformation gradient $F$ and the Green-Lagrange strain tensor $E$ :

$[\mathbf{F}]=\left[\begin{array}{ll}F_{11} & F_{12} \\ F_{21} & F_{22}\end{array}\right], E=\frac{1}{2}\left({ }^{T} F F-I\right)$

where $F_{11}=\frac{\partial}{\partial u}(D U(u, v)), F_{12}=\frac{\partial}{\partial v}(D U(u, v)), F_{22}=$ $\frac{\partial}{\partial v}(D V(u, v))$ and $F_{21}=\frac{\partial}{\partial u}(D V(u, v))$.

\section{Appendix B. Supplementary materials}

Supplementary data associated with this article can be found in the online version at doi:10.1016/j.jbiomech. 2005.12.010.

\section{References}

Cheng, P., Sutton, M.A., Schreier, H.W., McNeill, S.R., 2002. Fullfield speckle pattern image correlation with B-spline deformation function. Experimental Mechanics 42 (3), 344-352.

Choi, H.S., Vito, R.P., 1990. Two-dimensional stress-strain relationship for canine pericardium. Journal of Biomechanical Engineering 112, 153-159.

Lanir, Y., Fung, Y.C., 1974. Two-dimensional mechanical properties of rabbit skin-II. Experimental results. Journal of Biomechanics 7, 171-182.

Lee, J.B., Yang, K.H., 2002. Abdominal injury patterns in motor vehicle accidents: a survey of the NASS database from 1993 to 1997. Traffic Injury Prevention 3, 241-246.

Lu, H., Cary, P.D., 2000. Deformation measurements by digital image correlation: implementation of a second-order displacement gradient. Experimental Mechanics 40 (4), 393-400.

Marcellier, H., Vescovo, P., Varchon, D., Vacher, P., Humbert, P., 2001. Optical analysis of displacement and strain fields on human skin. Skin Research and Technology 7, 246-253. 
Mguil-Touchal, S., Morestin, F., Brunet, M., 1997. Various experimental applications of digital image correlation method. CMEM 97. Rhodes.

Mguil-Touchal, S., Morestin, F., Brunet, M., 1998. Experimental determination of forming limit diagrams using a method of digital picture. IDDRG 98. In: Proceedings of the 20th Biennal Congress. Genval Brussel.

Sacks, M.S., 1999. A method for planar biaxial mechanical testing that includes in-plane shear. Journal of Biomechanical Engineering 121, 551-555.

SAEJ211/1, Rev. Mach, 1995. Developed by the SAE Safety Test Instrumentation Standards Committee of the SAE Technical and Research Group. International, 400 Commonwealth Drive, Warrendale, Pennsylvania 15096.

Sanghavi, P., Bose, D., Kerrigan, J., Madeley, N.J., Crandall, J., 2004. Non-contact strain measurement of biological tissue. Biomedical Sciences Instrumentation 40, 51-56.

Schreier, H.W., Sutton, M.A., 2002. Systematic errors in digital image correlation due to undermatched subset shape functions. Experimental Mechanics 42 (3), 303-310.

Silver, F.H., Kato, Y.P., Ohno, M., Wasserman, A.J., 1992. Analysis of mammalian connective tissue: relationship between hierarchical structures and mechanical properties. Journal of Long-term Effects of Medical Implants 2, 165-198.
Snedeker, J.G., Niederer, P., Schmidlin, F.R., Farshad, M., Demetropoulos, C.K., Lee, J.B., Yang, K.H., 2005. Strain-rate dependent material properties of the porcine and human kidney capsule. Journal of Biomechanics 38, 1011-1021.

Stemper, B.D., Yoganandan, N., Pintar, F.A., 2005. Methodology to study intimal failure mechanics in human internal carotid arteries. Journal of Biomechanics 38 (12), 2491-2496.

Sutton, M.A., Wolters, W.J., Peters, W.H., Ranson, W.F., McNeill, S.R., 1983. Determination of displacements using an improved digital correlation method. Image and Vision Computing 1 (3), 133-139.

Sutton, M.A., Mingqi, C., Peters, W.H., Chao, Y.S., McNeill, S.R., 1986. Application of an optimized digital correlation method to planar deformation analysis. Image and Vision Computing 4 (3), $143-150$.

Yamada, H., 1970. Mechanical properties of the nervous system, integument, sense organs and tissues. In: Evans, G. (Ed.), Strength of Biological Materials. Waverly Press, Baltimore, pp. 224-231.

Yoganandan, N., Pintar, F.A., Gennarelli, T.A., 2000. Patterns of abdominal injuries in frontal and side impacts. In: Proceedings of the 44th Annual Scientific Conference of the AAAM. Chicago, Illinois. 